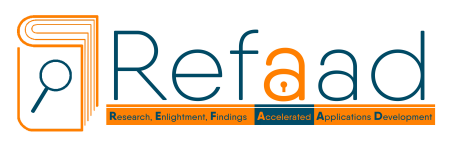

General Letters in Mathematics Vol. 3, No. 2, Oct 2017, pp.91-101

e-ISSN 2519-9277, p-ISSN 2519-9269

Available online at http:// www.refaad.com

\title{
Soft Fuzzy Metric Spaces
}

\author{
Ferhan Şola Erduran ${ }^{*}$, Ebru Yiğit ${ }^{2}$ \\ Rabia Alar ${ }^{3}$ and Ayten Gezici ${ }^{4}$ \\ 1,2,3,4 Gazi University, Department of Mathematics, 06500 Teknikokullar,Ankara,Turkey. \\ ${ }^{1}$ ferhansola@gazi.edu.tr, \\ 2 yigittebru@gmail.com.tr, ${ }^{3}$ rabiaalar76@gmail.com.tr, ${ }^{4}$ ayten.gezici.7@gmail.com.tr
}

\begin{abstract}
In this paper we introduced a new notion which is called soft fuzzy metric and examined some basic properties of soft fuzzy metric spaces. Besides, some topological structures of this new space such as; soft open and soft closed sets, soft interior and soft closure of a soft set are defined.
\end{abstract}

Keywords: Soft fuzzy metric, soft set, soft real number.

MSC2010 54A40,03E72.

\section{Introduction}

Generally we cannot successfully use classical methods for some problems because of various types of uncertainities. To solve this kinds of problems, there are theories such as; theory of probability [13, theory of fuzzy sets [16], theory of intuitionistic fuzzy sets [1] and theory of interval mathematics 2] which can be considered as mathematical tools for dealing with uncertainities. But these theories have some difficulties as pointed out in 12 . The reason for these difficulties is inadequency of the parametrization tool of the theories. For this reason, Molodtsov [12] introduced the concept of soft set theory as a mathematical tool for uncertainities which is free from difficulties. The soft set is a parametrized family of subsets of the universal set. The parameter set may be any collection of cohice, sentences, natural numbers etc. Hence, soft set theory has compelling applications in several diverse fields.

Research work in soft set theory have been progressing by Maji et al. [10, 11. They made a theoritical study of the soft set theory and presented an application of soft sets in a decision making problem. After that many authors studied in soft set theory and its application in various field [3, 9, 14, 15. Also, Das and Samanta [6, 7, 8, made contribution to this field by introducing a notion of soft metric space which is based on soft point of soft sets. Afterwards, Beaula and Gunaseli [4 defined fuzzy soft metric space in terms of fuzzy soft point and studied about its properties. In their definition a fuzzy soft metic $d$ is defined in two-dimensional space by using fuzzy soft point as $d: F S C\left(F_{E}\right) \times F S C\left(F_{E}\right) \rightarrow \mathbb{R}\left(A^{*}\right)$ [4. At the same time Beaula and Priyanga [5] gave a new notion for fuzzy soft normed linear space. They mainly studied about fuzzy soft normed space but also gave only a definition which is also called fuzzy soft metric space, is as follows:

A mapping $\Delta: S S P(\tilde{X}) \times S S P(\tilde{X}) \times \mathbb{R}\left(A^{*}\right) \rightarrow[0,1]$ is said to be a fuzzy soft metric on the soft set $\tilde{X}$ if $\Delta$ satisfies the following conditions:

1. $\Delta\left(\tilde{x}_{e_{1}}, \tilde{y}_{e_{2}}, \tilde{t}\right)=0$, if $\tilde{t}=\tilde{0}$,

2. $\Delta\left(\tilde{x}_{e_{1}}, \tilde{y}_{e_{2}}, \tilde{t}\right)=1$, for all $\tilde{t} \tilde{>} \tilde{0}$ if and only if $\tilde{x}_{e_{1}}=\tilde{y}_{e_{2}}$,

\footnotetext{
${ }^{*}$ Corresponding author. Ferhan Şola Erduran ${ }^{1}$ ferhansola@gazi.edu.tr
} 
3. $\Delta\left(\tilde{x}_{e_{1}}, \tilde{y}_{e_{2}}, \tilde{t}\right)=\Delta\left(\tilde{y}_{e_{2}}, \tilde{x}_{e_{1}}, \tilde{t}\right)$,

4. $\Delta\left(\tilde{x}_{e_{1}}, \tilde{z}_{e_{3}}, \tilde{s} \oplus \tilde{t}\right) \tilde{\geq} \Delta\left(\tilde{x}_{e_{1}}, \tilde{y}_{e_{2}}, \tilde{s}\right) * \Delta\left(\tilde{y}_{e_{2}}, \tilde{z}_{e_{3}}, \tilde{t}\right)$ for all $\tilde{t}, \tilde{s} \tilde{>} \tilde{0}$,

5. $\Delta\left(\tilde{x}_{e_{1}}, \tilde{y}_{e_{2}},.\right):(0, \infty) \rightarrow[0,1]$ is continuous.

The soft set $\tilde{X}$ with a fuzzy soft metric $\Delta$ is called a fuzzy soft metric space and denoted by $(\tilde{X}, \Delta, *)$.

Consequently the mappings $d$ and $\Delta$ mentioned above, don't have same structure but they have same name.

In this paper we give a new definition which is called soft fuzzy metric spaces, by using soft points of soft sets and soft real numbers of soft real sets. This new notion is so different from the definitions of fuzzy soft metric. For this notion we give definition soft t-norm $\tilde{*}$ for the triangle inequality of soft fuzzy metric and give some properties of soft t-norm $\tilde{*}$. Note that one of the main difference from fuzzy soft metric is to be the range of soft fuzzy metric on interval $[0,1](E)$ which means soft real numbers in $[0,1]$. After that, the structure of this new space is examined, some illustrate examples are given.

\section{Preliminaries}

Throughout this paper $X$ denotes initial universe set, $E$ denotes the set of all possible parameters which are attributes, characteristic or properties of the objects in $X$ and the set of all subsets of $X$ will be denoted by $P(X)$.

Definition 2.1. [12] Let $X$ be the initial universe set and $E$ be the set of parameters. A pair $(F, E)$ or $F_{E}$ is called a soft set over $X$, where $F$ is mapping given by $F: E \rightarrow P(X)$. In other words, the soft set is a parameterized of subsets of the set $X$. For $e \in E, F(e)$ may be considered as the set of e-elements of the soft set $(F, E)$ or as the set of e-approximate elements of the soft set of $(F, E)$.

Definition 2.2. [3] Let $X$ be the initial universe set, $E$ be the set of parameters and $A \subset E$. Then the mapping $F: E \rightarrow P(X)$ defined by, $e \in A, F(e) \neq \varnothing, e \notin A, F(e)=\varnothing$.

Definition 2.3. [3] The complement of a soft set $F$ is denoted by $F^{c}$, where $F^{c}: E \rightarrow P(X)$ is a mapping given by $F^{c}(e)=X-F(e)$, for all $e \in E$.

Definition 2.4. [11] A soft set $(F, E)$ over $X$ is said to be an absolute soft set denoted by $\tilde{X}$ if for all $e \in$ $E, F(e)=X$.

Definition 2.5. [11] A soft set $(F, E)$ over $X$ is said to be a null soft set denoted by $\Phi$ if for all $e \in E, F(e)=\varnothing$.

Definition 2.6. 77 Let $X$ be non-empty set and $E$ be a non-empty parameter set. Then a function $\varepsilon: E \rightarrow X$ is said to be soft element of $X$. A soft element $\varepsilon$ of $X$ is said to belongs to a soft set $A$ of $X$, which is denoted by $\varepsilon \tilde{\in} A$, if $\varepsilon(e) \in A(e), \forall e \in E$. Thus for a soft set $A$ of $X$ with respect to the index set $E$, we have $A(e)=\{\varepsilon(e), \varepsilon \tilde{\in} A\}, e \in E$.

It is to be noted that every singleton soft set (a soft set $(F, E)$ for which $F(e)$ is a singleton set, $\forall e \in E$ ) can be identified with a soft element by simply identifying the singleton set with the element that it contains for all $e \in E$.

Definition 2.7. [7] Let $\mathbf{R}$ be the set of real numbers, $\mathfrak{B}(\mathbf{R})$ the collection of all non-empty bounded subsets of $\mathbf{R}$ and $E$ a set of parameters. Then a mapping $F: E \rightarrow \mathfrak{B}(\mathbf{R})$ is called a soft real set. It is denoted by $(F, E)$ and $\mathbf{R}(E)$ denotes the set of all soft real sets. Also, $\mathbf{R}(E)^{*}$ denotes the set of all non-negative soft real sets $((F, E)$ is said to be a non-negative soft real set if $F(\lambda)$ is a subset of the set of non-negative real numbers for each $\lambda \in E)$.

In particular, if $(F, E)$ is a singleton soft set, then identifying $(F, E)$ with the corresponding soft element, we will call it a soft real number. $R(E)$ denotes the set of all soft real numbers. We use notations $\tilde{r}, \tilde{s}, \tilde{t}$ to denote soft real numbers.

Definition 2.8. [8] For two soft real numbers $\tilde{r}, \tilde{s} \in R(E)$ we define

1. $\tilde{r} \tilde{\leq} \tilde{s}$ if $\tilde{r}(\lambda) \leq \tilde{s}(\lambda)$, for all $\lambda \in E$;

2. $\tilde{r} \tilde{\geq} \tilde{s}$ if $\tilde{r}(\lambda) \geq \tilde{s}(\lambda)$, for all $\lambda \in E$;

3. $\tilde{r} \tilde{<} \tilde{s}$ if $\tilde{r}(\lambda)<\tilde{s}(\lambda)$, for all $\lambda \in E$;

4. $\tilde{r} \tilde{>} \tilde{s}$ if $\tilde{r}(\lambda)>\tilde{s}(\lambda)$, for all $\lambda \in E$. 
Definition 2.9. [15] For any two soft real numbers $\tilde{r}, \tilde{s} \in R(E)$,

1. Define the operation $\oplus, \ominus, \odot$ respectively on by : $(\tilde{r} \oplus \tilde{s})(e)=\{\{\tilde{r}(e)+\tilde{s}(e)\}, e \in E\},(\tilde{r} \ominus \tilde{s})(e)=\{\{\tilde{r}(e)-\tilde{s}(e)\}, e \in E\},(\tilde{r} \odot \tilde{s})(e)=\{\{r(e) . s(e)\}, e \in E\}$.

2. The addivite inverse of a soft real number $\tilde{r}$ is denoted by $-\tilde{r}$ and given by $(-\tilde{r})(e)=\{\{-\tilde{r}(e)\}, e \in E\}$.

3. The multiplication inverse of a soft real number which is totally different than $\tilde{0}$ is denoted by $\tilde{r}^{-1}$ and given by $\tilde{r}^{-1}(e)=\frac{1}{\tilde{r}(e)}=\left\{\left\{\frac{1}{\tilde{r}(e)}\right\}, e \in E\right\}$.

Example 2.10. Consider $E=\left\{e_{1}, e_{2}, e_{3}, e_{4}, e_{5}, e_{6}\right\}$ and let the soft real numbers $\tilde{r}, \tilde{l}, \tilde{m}$ given as :

$\tilde{r}=\left\{\left(e_{1}, 1\right),\left(e_{2},-3\right),\left(e_{3},-\frac{2}{5}\right),\left(e_{4}, 5\right),\left(e_{5}, 2\right),\left(e_{6}, 4\right)\right\}$,

$\tilde{l}=\left\{\left(e_{1}, \frac{1}{4}\right),\left(e_{2}, 4\right),\left(e_{3},-2\right),\left(e_{4},-3\right),\left(e_{5}, 6\right),\left(e_{6}, \frac{2}{3}\right)\right\}$,

$\tilde{m}=\left\{\left(e_{1}, 7\right),\left(e_{2},-1\right),\left(e_{3}, \frac{4}{7}\right),\left(e_{4}, 2\right),\left(e_{5}, 3\right),\left(e_{6}, 8\right)\right\}$.

We will see that $(\tilde{r} \oplus \tilde{l})\left(e_{1}\right)=1+\frac{1}{4}=\frac{5}{4}$ and $(\tilde{l} \odot \tilde{m})\left(e_{6}\right)=\frac{2}{3} \cdot 8=\frac{16}{3}$ and so on.

Remark 2.11. [7] $\bar{r}, \bar{s}, \bar{t}$ denote a particular type of soft real numbers such that $\bar{r}(\lambda)=r$, for all $\lambda \in E$. For example $\overline{0}$ is the soft real number, where $\overline{0}(\lambda)=0$, for all $\lambda \in E$.

Definition 2.12. [6] A soft set $(F, A)$ over $X$ is said to be a soft point, if there exist $x \in X$ and $e \in E$ such that $F(e)=\{x\}$ and $F\left(e^{\prime}\right)=\varnothing$ some $x \in X$ and $\forall e^{\prime} \in E \backslash\{e\}$. It will be denoted by $\tilde{x}_{e}$.

Definition 2.13. [6] A soft point $\tilde{x}_{e}$ is said to be belongs to a soft set $(G, A)$, if for the element e $\in E ; F(e)=$ $\{x\} \subset G(e)$. We write $\tilde{x}_{e} \tilde{\in}(G, A)$.

Definition 2.14. [6] Two soft points $\tilde{x}_{e_{1}}, \tilde{y}_{e_{2}}$ are said to be equal if $e_{1}=e_{2}$ and $x=y$. If $\tilde{x}_{e_{1}} \neq \tilde{y}_{e_{2}} \Leftrightarrow x \neq y$ or $e_{1} \neq e_{2}$.

[6] The union of any collection of soft points can be considered as a soft set and every soft set can be expressed as union of all soft points belonging to it; i.e., $(F, A)=\bigcup_{\tilde{x}_{e} \tilde{\epsilon}(F, A)} \tilde{x}_{e}$.

Remark 2.15. [6] If $\mathfrak{B}$ be a collections of soft points, then the soft set generated by taking all the soft points of $\mathfrak{B}$ will be denoted by $S S(\mathfrak{B})$, whereas the collection of all soft points of a soft set $(F, A)$ will be expressed by $S P(F, A)$.

[6] For the collections $\mathfrak{B}, \mathfrak{B}_{1}$ and $\mathfrak{B}_{2}$ of soft points and for the soft sets $(F, A),(G, A)$;

1. $S P(S S(\mathfrak{B}))=\mathfrak{B}, S S(S P(F, A))=(F, A)$;

2. $S P((F, A) \tilde{\cup}(G, A))=S P(F, A) \cup S P(G, A)$ and $S P((F, A) \tilde{\cap}(G, A))=S P(F, A) \cap S P(G, A) ;$

3. $S S\left(\mathfrak{B}_{1} \cup \mathfrak{B}_{2}\right)=S S\left(\mathfrak{B}_{1}\right) \tilde{\cup} S\left(\mathfrak{B}_{2}\right)$ and $S S\left(\mathfrak{B}_{1} \cap \mathfrak{B}_{2}\right)=S S\left(\mathfrak{B}_{1}\right) \tilde{\cap} S S\left(\mathfrak{B}_{2}\right)$.

\section{Soft Fuzzy Metric Space}

In this section, we define soft fuzzy metric by using soft points by the help of soft $t$-norm $\tilde{*}$ and give some properties of soft $t$-norms and soft fuzzy metric spaces.

Let $X$ be a non-empty set and $E$ be the nonempty set of parameters. Let $\tilde{X}$ be an absolute soft set, $S P(\tilde{X})$ be the collection of all soft points of $\tilde{X}$ and $\mathbb{R}(E)^{*}$ denote the set of all non-negative soft real numbers. Soft real numbers in the $[0,1]$ and $(0, \infty)$ are indicated by $[0,1](E)$ and $(0, \infty)(E)$ respectively.

Definition 3.1. A soft fuzzy set $S$ in $\tilde{X}$ is a set of ordered pairs:

$$
S=\left\{\left(\tilde{x}_{e}, \mu_{s}\left(\tilde{x}_{e}\right)\right): \tilde{x}_{e} \in \tilde{X}, e \in E\right\}
$$

where $\mu_{s}: \tilde{X} \rightarrow[0,1](E)$ is called the soft membership function and $\mu_{s}\left(\tilde{x}_{e}\right)$ is grade of soft membership of $\tilde{x}_{e}$ in $S$. 
Definition 3.2. Let $\tilde{*}:[0,1](E) \times[0,1](E) \rightarrow[0,1](E) . \tilde{*}$ is called continuous soft $t$-norm if $\tilde{*}$ satisfying the following conditions:

i. $\tilde{*}$ is commutative and associativite;

ii. $\tilde{*}$ is continuous;

iii. $\tilde{a} \tilde{*} \overline{1}=\tilde{a}$, for all $\tilde{a} \tilde{\in}[0,1](E)$;

iv. $\tilde{a} \tilde{a} \tilde{b} \tilde{\leq} \tilde{c} \tilde{*} \tilde{d}$, whenever $\tilde{a} \tilde{\leq} \tilde{c}$ and $\tilde{b} \tilde{\leq} \tilde{d}$ and $\tilde{a}, \tilde{b}, \tilde{c}, \tilde{d} \tilde{\epsilon}[0,1](E)$.

Example 3.3. Let $E=\left\{e_{1}, e_{2}, e_{3}, e_{4}\right\} .(\tilde{a} \tilde{*} \tilde{b})(e)=(\tilde{a} \odot \tilde{b})(e)($ for short $(\tilde{a} \tilde{*} \tilde{b})=\tilde{a} \odot \tilde{b})$ is a continous soft t-norm. We show that $\tilde{*}$ satisfies all of the above conditions. $i) \tilde{a} \tilde{*}(\tilde{b} \tilde{*} \tilde{c})=\tilde{a} \odot(\tilde{b} \tilde{*} \tilde{c})=\tilde{a} \odot(\tilde{b} \odot \tilde{c})=(\tilde{a} \odot \tilde{b}) \odot \tilde{c}=(\tilde{a} \tilde{*} \tilde{b}) \odot \tilde{c}=$ $(\tilde{a} \tilde{*} \tilde{b}) \tilde{*} \tilde{c}$,

$\tilde{a} \tilde{*} \tilde{b}=\tilde{a} \odot \tilde{b}=\tilde{b} \odot \tilde{a}=\tilde{b} \tilde{*} \tilde{a}$.

ii) It is obvious.

iii) $\tilde{a} \tilde{*} \overline{1}=\tilde{a} \odot \overline{1}=\tilde{a}$, for all $\tilde{a} \tilde{\in}[0,1](E)$.

iv Let $\tilde{a} \tilde{\leq} \tilde{c}$ and $\tilde{b} \tilde{\leq} \tilde{d}$, then $\tilde{a} \tilde{*} \tilde{b}=\tilde{a} \odot \tilde{b} \leq \tilde{c} \odot \tilde{d}=\tilde{c} \tilde{*} \tilde{d}$.

For example, we calculate $\tilde{a} \tilde{*} \tilde{b}$ for $\tilde{a}=\left\{\left(e_{1}, \frac{1}{2}\right),\left(e_{2}, \frac{3}{4}\right),\left(e_{3}, 1\right)\right\}$ and $\tilde{b}=\left\{\left(e_{1}, \frac{1}{5}\right),\left(e_{2}, \frac{3}{5}\right),\left(e_{3}, 0\right)\right\}$.

$(\tilde{a} \tilde{*} \tilde{b})(e)=(\tilde{a} \odot \tilde{b})(e)=\tilde{a}(e) \cdot \tilde{b}(e)$ for all $e \in E$.

$$
\begin{aligned}
\tilde{a}\left(e_{1}\right) & =\frac{1}{2}, \tilde{b}\left(e_{1}\right)=\frac{1}{5} \Rightarrow \tilde{a}\left(e_{1}\right) \cdot \tilde{b}\left(e_{1}\right)=\frac{1}{10} \\
\tilde{a}\left(e_{2}\right) & =\frac{3}{4}, \tilde{b}\left(e_{2}\right)=\frac{3}{5} \Rightarrow \tilde{a}\left(e_{2}\right) \cdot \tilde{b}\left(e_{2}\right)=\frac{9}{20} \\
\tilde{a}\left(e_{3}\right) & =1, \tilde{b}\left(e_{3}\right)=0 \Rightarrow \tilde{a}\left(e_{3}\right) \cdot \tilde{b}\left(e_{3}\right)=0 \\
\tilde{a} \tilde{*} \tilde{b} & =\left\{\left(e_{1}, \frac{1}{10}\right),\left(e_{2}, \frac{9}{20}\right),\left(e_{3}, 0\right)\right\} .
\end{aligned}
$$

Also, it can easily be shown that $\tilde{a} \tilde{*} \tilde{b}=\min \{\tilde{a}, \tilde{b}\}$ and $\tilde{a} \tilde{a} \tilde{b}=\max \{\overline{0}, \tilde{a} \oplus \tilde{b}-\overline{1}\}$ are continuous soft $t$-norms for all $\tilde{a}, \tilde{b} \tilde{\epsilon}[0,1](E)$.

Remark 3.4. $\tilde{*}$ satisfies the following conditions:

i. For any $\tilde{r}_{1}, \tilde{r}_{2} \tilde{\in}(0,1)(E)$ with $\tilde{r}_{1} \tilde{>} \tilde{r}_{2}$, there exists $\tilde{r}_{3} \tilde{\in}(0,1)(E)$ such that $\tilde{r}_{1} \tilde{*} \tilde{r}_{3} \tilde{\geq} \tilde{r}_{2}$.

ii. For any $\tilde{r}_{4} \tilde{\in}(0,1)(E)$, there exists $\tilde{r}_{5} \tilde{\in}(0,1)(E)$ such that $\tilde{r}_{4} \tilde{*} \tilde{r}_{4} \tilde{\geq} \tilde{r}_{5}$.

Definition 3.5. A mapping $S: S P(\tilde{X}) \times S P(\tilde{X}) \times(0, \infty)(E) \rightarrow[0,1](E)$ is said to be a soft fuzzy metric on the soft set $\tilde{X}$, if $S$ satisfies the following conditions:

$(S F M-1) S\left(\tilde{x}_{e_{1}}, \tilde{y}_{e_{2}}, \tilde{t}\right) \tilde{>} \overline{0}$, for all $\tilde{x}_{e_{1}}, \tilde{y}_{e_{2}} \tilde{\in} \tilde{X}$ and $\tilde{t} \tilde{>} \overline{0}$,

(SFM-2) $S\left(\tilde{x}_{e_{1}}, \tilde{y}_{e_{2}}, \tilde{t}\right)=\overline{1}$ if and only if $\tilde{x}_{e_{1}}=\tilde{y}_{e_{2}}$, for all $\tilde{x}_{e_{1}}, \tilde{y}_{e_{2}} \tilde{\in} \tilde{X}$ and $\tilde{t} \tilde{>} \overline{0}$,

$(S F M-3) S\left(\tilde{x}_{e_{1}}, \tilde{y}_{e_{2}}, \tilde{t}\right)=S\left(\tilde{y}_{e_{2}}, \tilde{x}_{e_{1}}, \tilde{t}\right)$, for all $\tilde{x}_{e_{1}}, \tilde{y}_{e_{2}} \tilde{\in} \tilde{X}$ and $\tilde{t} \tilde{>} \overline{0}$,

$(S F M-4) S\left(\tilde{x}_{e_{1}}, \tilde{z}_{e_{3}}, \tilde{t} \oplus \tilde{s}\right) \tilde{\geq} S\left(\tilde{x}_{e_{1}}, \tilde{y}_{e_{2}}, \tilde{t}\right) \tilde{*} S\left(\tilde{y}_{e_{2}}, \tilde{z}_{e_{3}}, \tilde{s}\right)$, for all $\tilde{x}_{e_{1}}, \tilde{y}_{e_{2}}, \tilde{z}_{e_{3}} \tilde{\in} \tilde{X}$ and $\tilde{t} \tilde{>} \overline{0}$,

$(S F M-5) S\left(\tilde{x}_{e_{1}}, \tilde{y}_{e_{2}},.\right):(0, \infty)(E) \rightarrow[0,1](E)$ is continuous.

The soft set $\tilde{X}$ with a soft fuzzy metric $S$ on $\tilde{X}$ is called a soft fuzzy metric space and denoted by $(\tilde{X}, S, \tilde{*})$.

Example 3.6. Let $X \subset R$ be a non-empty set and $E \subset R$ be the non-empty set of parameters. Let $\tilde{X}$ be the absolute soft set and $\bar{x}$ denote the soft real number such that $\bar{x}(e)=x$, for all $e \in E$. In the example (4.3) of [G] the function $d: S P(\tilde{X}) \times S P(\tilde{X}) \rightarrow \mathbb{R}(E)^{*}$ defined by $d\left(\tilde{x}_{e_{1}}, \tilde{y}_{e_{2}}\right)=|\bar{x} \ominus \bar{y}|+\left|e_{1}-e_{2}\right|$, for all $\tilde{x}_{e_{1}}, \tilde{y}_{e_{2}} \tilde{\in} \tilde{X}$, then $d$ is a soft metric on $\tilde{X}$. Define the fuction $S: S P(\tilde{X}) \times S P(\tilde{X}) \times(0, \infty)(E) \rightarrow[0,1](E)$ by

$$
S\left(\tilde{x}_{e_{1}}, \tilde{y}_{e_{2}}, \tilde{t}\right)=\frac{\tilde{t}}{\tilde{t} \oplus d\left(\tilde{x}_{e_{1}}, \tilde{y}_{e_{2}}\right)},
$$


for all $\tilde{x}_{e_{1}}, \tilde{y}_{e_{2}} \tilde{\in} \tilde{X}, \tilde{t} \tilde{>} \overline{0}$ and $\tilde{a} \tilde{*} \tilde{b}=\min \{\tilde{a}, \tilde{b}\}$ for all $\tilde{a}, \tilde{b} \tilde{\in}[0,1](E)$. Then it can be easily verified that $S$ is a soft fuzzy metric on $\tilde{X}$.

Definition 3.7. A mapping $S: S P(\tilde{X}) \times S P(\tilde{X}) \times(0, \infty)(E) \rightarrow[0,1](E)$ is said to be a soft fuzzy pseudo-metric on the soft set $\tilde{X}$ if $S$ satisfies the following conditions:

$(p-1) S\left(\tilde{x}_{e_{1}}, \tilde{y}_{e_{2}}, \tilde{t}\right) \tilde{>} \overline{0}$, for all $\tilde{x}_{e_{1}}, \tilde{y}_{e_{2}} \tilde{\in} \tilde{X}$ and $\tilde{t} \tilde{>} \overline{0}$

(p-2) $S\left(\tilde{x}_{e_{1}}, \tilde{y}_{e_{2}}, \tilde{t}\right)=\overline{1}$ if $\tilde{x}_{e_{1}}=\tilde{y}_{e_{2}}$, for all $\tilde{x}_{e_{1}}, \tilde{y}_{e_{2}} \tilde{\in} \tilde{X}$ and $\tilde{t} \tilde{>} \overline{0}$,

$(p-3) S\left(\tilde{x}_{e_{1}}, \tilde{y}_{e_{2}}, \tilde{t}\right)=S\left(\tilde{y}_{e_{2}}, \tilde{x}_{e_{1}}, \tilde{t}\right)$, for all $\tilde{x}_{e_{1}}, \tilde{y}_{e_{2}} \tilde{\in} \tilde{X}$ and $\tilde{t} \sim \overline{0}$,

$(p-4) S\left(\tilde{x}_{e_{1}}, \tilde{z}_{e_{3}}, \tilde{t} \oplus \tilde{s}\right) \tilde{\geq} S\left(\tilde{x}_{e_{1}}, \tilde{y}_{e_{2}}, \tilde{t}\right) \tilde{*} S\left(\tilde{y}_{e_{2}}, \tilde{z}_{e_{3}}, \tilde{s}\right)$, for all $\tilde{x}_{e_{1}}, \tilde{y}_{e_{2}}, \tilde{z}_{e_{3}} \tilde{\in} \tilde{X}$ and $\tilde{t} \tilde{>} \overline{0}$,

$(p-5) S\left(\tilde{x}_{e_{1}}, \tilde{y}_{e_{2}},.\right):(0, \infty)(E) \rightarrow[0,1](E)$ is continuous.

The soft set $\tilde{X}$ with a soft fuzzy pseudo-metric $S$ on $\tilde{X}$ is called a soft fuzzy pseudo-metric space and denoted by $(\tilde{X}, S, \tilde{*})$.

Example 3.8. Let $\tilde{X}$ be the absolute soft set over the non-empty set of parameters E. From the example (4.3)

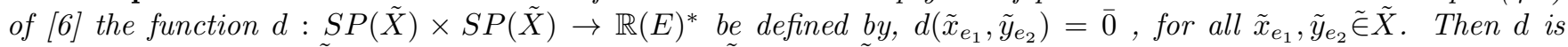
soft-pseudo metric on $\tilde{X}$. Define the fuction $S: S P(\tilde{X}) \times S P(\tilde{X}) \times(0, \infty)(E) \rightarrow[0,1](E)$ by,

$$
S\left(\tilde{x}_{e_{1}}, \tilde{y}_{e_{2}}, \tilde{t}\right)=\frac{\tilde{t}}{\tilde{t} \oplus d\left(\tilde{x}_{e_{1}}, \tilde{y}_{e_{2}}\right)},
$$

for all $\tilde{x}_{e_{1}}, \tilde{y}_{e_{2}} \tilde{\in} \tilde{X}$ and $\tilde{t} \sim \overline{0}$ and denote $\tilde{a} \tilde{*} \tilde{b}=\min \{\tilde{a}, \tilde{b}\}$ for all $\tilde{a}, \tilde{b} \tilde{\in}[0,1](E)$. Then it can be easily verified that $S$ is a soft fuzzy pseudo-metric on $\tilde{X}$.

Definition 3.9. Let $(\tilde{X}, S, \tilde{*})$ be soft fuzzy metric space, $\tilde{r} \tilde{\in}(0,1)(E)$ and $\tilde{t} \tilde{>} \overline{0}$. For any $\tilde{x}_{e_{1}} \tilde{\in} \tilde{X}$ by an open ball with centre $\tilde{x}_{e_{1}}$ and radius $\tilde{r}$, we mean the collection of soft points of $\tilde{X}$ satisfying $S\left(\tilde{x}_{e_{1}}, \tilde{y}_{e_{2}}, \tilde{t}\right) \tilde{>} \overline{1} \ominus \tilde{r}$. The open ball with centre $\tilde{x}_{e_{1}}$ and radius $\tilde{r}$ with respect to $\tilde{t}$ is denoted by $B_{S}\left(\tilde{x}_{e_{1}}, \tilde{r}, \tilde{t}\right)$.

That is, $B_{S}\left(\tilde{x}_{e_{1}}, \tilde{r}, \tilde{t}\right)=\left\{\tilde{y}_{e_{2}}: S\left(\tilde{x}_{e_{1}}, \tilde{y}_{e_{2}}, \tilde{t}\right) \tilde{>} \overline{1} \ominus \tilde{r}\right\} \subset S P(\tilde{X}) . S S\left(B_{S}\left(\tilde{x}_{e_{1}}, \tilde{r}, \tilde{t}\right)\right)$ will be called a soft open ball with centre $\tilde{x}_{e_{1}}$ and radius $\tilde{r}$ with respect to $\tilde{t}$.

Note that $B_{S}\left(\tilde{x}_{e_{1}}, \tilde{r}, \tilde{t}\right)$ is a collection of soft points on $\tilde{X}$ and $S S\left(B_{S}\left(\tilde{x}_{e_{1}}, \tilde{r}, \tilde{t}\right)\right)$ is a soft set on $\tilde{X}$.

Definition 3.10. Let $(\tilde{X}, S, \tilde{*})$ be soft fuzzy metric space, $\tilde{r} \tilde{\epsilon}(0,1)(E)$ and $\tilde{t} \tilde{>} \overline{0}$. For any $\tilde{x}_{e_{1}} \tilde{\in} \tilde{X}$ by a closed ball with centre $\tilde{x}_{e_{1}}$ and radius $\tilde{r}$, we mean the collection of soft points of $\tilde{X}$ satisfying $S\left(\tilde{x}_{e_{1}}, \tilde{y}_{e_{2}}, \tilde{t}\right) \tilde{\geq} \overline{1} \ominus \tilde{r}$. The closed ball with centre $\tilde{x}_{e_{1}}$ and radius $\tilde{r}$ with respect to $\tilde{t}$ is denoted by $B_{S}\left[\tilde{x}_{e_{1}}, \tilde{r}, \tilde{t}\right]$.

That is, $B_{S}\left[\tilde{x}_{e_{1}}, \tilde{r}, \tilde{t}\right]=\left\{\tilde{y}_{e_{2}}: S\left(\tilde{x}_{e_{1}}, \tilde{y}_{e_{2}}, \tilde{t}\right) \tilde{\geq} \overline{1} \ominus \tilde{r}\right\} \subset S P(\tilde{X}) . S S\left(B_{S}\left[\tilde{x}_{e_{1}}, \tilde{r}, \tilde{t}\right]\right)$ will be called a soft closed ball with centre $\tilde{x}_{e_{1}}$ and radius $\tilde{r}$ with respect to $\tilde{t}$.

Note that $B_{S}\left[\tilde{x}_{e_{1}}, \tilde{r}, \tilde{t}\right]$ is a collection of soft points on $\tilde{X}$ and $S S\left(B_{S}\left[\tilde{x}_{e_{1}}, \tilde{r}, \tilde{t}\right]\right)$ is a soft set on $\tilde{X}$.

Definition 3.11. Let $(\tilde{X}, S, \tilde{*})$ be a soft fuzzy metric space, $(Y, A)$ be a non-empty soft subset of $\tilde{X}$ and $\tilde{*}$ be continuous soft t-norm. Then the mapping $S_{Y}: S P(Y, A) \times S P(Y, A) \times(0, \infty)(A) \rightarrow[0,1](A)$ defined by

$$
S_{Y}\left(\tilde{x}_{e_{1}}, \tilde{y}_{e_{2}}, \tilde{t}\right)=S\left(\tilde{x}_{e_{1}}, \tilde{y}_{e_{2}}, \tilde{t}\right)
$$

for all $\tilde{x}_{e_{1}}, \tilde{y}_{e_{2}} \tilde{\in}(Y, A)$ is a soft fuzzy metric on $(Y, A)$. This soft fuzzy metric is known as the relative soft fuzzy metric induced on $(Y, A)$ by $S$. The soft fuzzy metric space $\left((Y, A), S_{Y}, \tilde{*}\right)$ is called a subspace of the soft fuzzy metric space $(\tilde{X}, S, \tilde{*})$.

Definition 3.12. Let $(\tilde{X}, S, \tilde{*})$ be a soft fuzzy metric space having at least two soft points. Then $(\tilde{X}, S, \tilde{*})$ is said to have Hausdorff property, if $\tilde{x}_{e_{1}}, \tilde{y}_{e_{2}}$ in $\tilde{X}$ with $S\left(\tilde{x}_{e_{1}}, \tilde{y}_{e_{2}}, \tilde{t}\right) \tilde{<} \overline{1}$, then there are two soft open balls $S S\left(B B_{S}\left(\tilde{x}_{e_{1}}, \tilde{r}, \tilde{t}\right)\right)$ and $S S\left(B_{S}\left(\tilde{y}_{e_{2}}, \tilde{r}, \tilde{t}\right)\right)$ with centre $\tilde{x}_{e_{1}}$ and $\tilde{y}_{e_{2}}$ respectively and radius $\tilde{r} \tilde{\in}(0,1)(E)$ with respect to $\tilde{t}$ such that

$$
S S\left(B_{S}\left(\tilde{x}_{e_{1}}, \tilde{r}, \tilde{t}\right)\right) \tilde{\cap} S S\left(B_{S}\left(\tilde{y}_{e_{2}}, \tilde{r}, \tilde{t}\right)\right)=\tilde{\varnothing} .
$$


Theorem 3.13. Every soft fuzzy metric space is Hausdorff.

Proof: Let $(\tilde{X}, S, \tilde{*})$ be a soft fuzzy metric space and $\tilde{x}_{e_{1}}, \tilde{y}_{e_{2}}$ be two distinct points of $\tilde{X}$. Take $S\left(\tilde{x}_{e_{1}}, \tilde{y}_{e_{2}}, \tilde{t}\right)=\tilde{r}$ for some $\tilde{r}, \overline{0} \tilde{<} \tilde{r} \tilde{<} \tilde{1}$. For each $r_{0}, \tilde{r} \tilde{<} \tilde{r}_{0} \tilde{<} \overline{1}$, we can find $\tilde{r}_{1}$ such that $\tilde{r}_{1} \tilde{*} \tilde{r}_{1} \tilde{\geq}_{0}$. Now consider the soft open ball

$$
S S\left(B_{S}\left(\tilde{x}_{e_{1}}, \overline{1} \ominus \tilde{r}_{1}, \frac{\overline{1}}{2} \tilde{t}\right)\right)=S S\left(\left\{\tilde{k}_{e_{4}} \tilde{\in} \tilde{X}: S\left(\tilde{k}_{e_{4}}, \tilde{x}_{e_{1}}, \frac{\overline{1}}{2} \tilde{t}\right) \tilde{>}_{1}\right\}\right)
$$

and

We claim that

$$
S S\left(B_{S}\left(\tilde{y}_{e_{2}}, \overline{1} \ominus \tilde{r}_{1}, \frac{\overline{1}}{2} \tilde{t}\right)\right)=S S\left(\left\{\tilde{m}_{e_{5}} \tilde{\in} \tilde{X}: S\left(\tilde{m}_{e_{5}}, \tilde{y}_{e_{2}}, \frac{\overline{1}}{2} \tilde{t}\right) \tilde{>} \tilde{r}_{1}\right\}\right)
$$

$$
S S\left(B_{S}\left(\tilde{x}_{e_{1}}, \overline{1} \ominus \tilde{r}_{1}, \frac{\overline{1}}{2} \tilde{t}\right)\right) \tilde{\cap} S S\left(B_{S}\left(\tilde{y}_{e_{2}}, \overline{1} \ominus \tilde{r}_{1}, \frac{\overline{1}}{2} \tilde{t}\right)\right)=\Phi .
$$

Suppose that let

$$
S S\left(B_{S}\left(\tilde{x}_{e_{1}}, \overline{1} \ominus \tilde{r}_{1}, \frac{\overline{1}}{2} \tilde{t}\right)\right) \tilde{\cap} S S\left(B_{S}\left(\tilde{y}_{e_{2}}, \overline{1} \ominus \tilde{r}_{1}, \frac{\overline{1}}{2} \tilde{t}\right)\right) \neq \Phi .
$$

Then

and

$$
\tilde{z}_{e_{3}} \tilde{\in} S S\left(B_{S}\left(\tilde{x}_{e_{1}}, \overline{1} \ominus \tilde{r}_{1}, \frac{\overline{1}}{2} \tilde{t}\right)\right) \Rightarrow S\left(\tilde{x}_{e_{1}}, \tilde{z}_{e_{3}}, \frac{\overline{1}}{2} \tilde{t}\right) \tilde{>} \tilde{r}_{1}
$$

$$
\tilde{z}_{e_{3}} \tilde{\in} S S\left(B_{S}\left(\tilde{y}_{e_{2}}, \overline{1} \ominus \tilde{r}_{1}, \frac{\overline{1}}{2} \tilde{t}\right)\right) \Rightarrow S\left(\tilde{y}_{e_{2}}, \tilde{z}_{e_{3}}, \frac{\overline{1}}{2} \tilde{t}\right) \tilde{r}_{1}
$$

This means that

$$
\begin{aligned}
\tilde{r} & =S\left(\tilde{x}_{e_{1}}, \tilde{y}_{e_{2}}, \tilde{t}\right) \\
& \tilde{\geq} \quad S\left(\tilde{x}_{e_{1}}, \tilde{z}_{e_{3}}, \frac{\overline{1}}{2} \tilde{t}\right) * S\left(\tilde{z}_{e_{3}}, \tilde{y}_{e_{2}}, \frac{\overline{1}}{2} \tilde{t}\right) \\
& \tilde{>} \tilde{r}_{1} \tilde{*} \tilde{r}_{1} \tilde{\geq}_{0} \tilde{r}_{0} \tilde{r}
\end{aligned}
$$

which is contradiction. Therefore $(\tilde{X}, S, \tilde{*})$ is Hausdorff.

Definition 3.14. Let $(\tilde{X}, S, \tilde{*})$ be a soft fuzzy metric space and $\tilde{x}_{e_{1}} \tilde{\in} \tilde{X}$. A collection $N\left(\tilde{x}_{e_{1}}\right)$ of soft points containing the soft point $\tilde{x}_{e_{1}}$ is said to be neighbourhood of the soft point $\tilde{x}_{e_{1}}$, if there exist $\tilde{r} \tilde{\in}(0,1)(E)$ and $\tilde{t} \sim \overline{0}$ such that $\tilde{x}_{e_{1}} \in B_{s}\left(\tilde{x}_{e_{1}}, \tilde{r}, \tilde{t}\right) \subset N\left(\tilde{x}_{e_{1}}\right)$.

$S S\left(N\left(\tilde{x}_{e_{1}}\right)\right)$ will be called a soft neighbourhood of the soft point $\tilde{x}_{e_{1}}$.

Theorem 3.15. Let $(\tilde{X}, S, \tilde{*})$ be a soft fuzzy metric space and $\tilde{x}_{e_{1}} \in \tilde{X}$. Let $N_{1}$ and $N_{2}$ be neighbourhood of $\tilde{x}_{e_{1}}$ in $(\tilde{X}, S, \tilde{*})$. Then $S S\left(N_{1}\right) \tilde{\cap} S S\left(N_{2}\right)$ is a soft neighbourhood of $\tilde{x}_{e_{1}}$ in $(\tilde{X}, S, \tilde{*})$.

Proof: Let $(\tilde{X}, S, \tilde{*})$ be a soft fuzzy metric space, $\tilde{x}_{e_{1}} \in \tilde{X}, N_{1}, N_{2}$ be neighbourhood of $\tilde{x}_{e_{1}}$.

$N_{1}$ is a neighbourhood of $\tilde{x}_{e_{1}}$,

$\Rightarrow \exists \tilde{r}_{1} \tilde{\in}(0,1)(E)$ and $\tilde{t} \sim \overline{0}$ such that $\tilde{x}_{e_{1}} \in B_{s}\left(\tilde{x}_{e_{1}}, \tilde{r}_{1}, \tilde{t}_{1}\right) \subset N_{1}$

$\Rightarrow \tilde{x}_{e_{1}} \tilde{\in} S S\left(B_{s}\left(\tilde{x}_{e_{1}}, \tilde{r}_{1}, \tilde{t}_{1}\right)\right) \tilde{\subset} S S\left(N_{1}\right)$

$\Rightarrow \tilde{x}_{e_{1}} \tilde{\in} S S\left(\left\{\tilde{y}_{e_{2}}: S\left(\tilde{x}_{e_{1}}, \tilde{y}_{e_{2}}, \tilde{t}_{1}\right) \tilde{>} \overline{1} \ominus \tilde{r}_{1}\right\}\right) \tilde{\subset} S S\left(N_{1}\right)$.

$N_{2}$ is a neighbourhood of $\tilde{x}_{e_{1}}$,

$\Rightarrow \exists \tilde{r}_{2} \tilde{\in}(0,1)(E)$ and $\tilde{t} \tilde{>} \overline{0}$ such that $\tilde{x}_{e_{1}} \in B_{s}\left(\tilde{x}_{e_{1}}, \tilde{r}_{2}, \tilde{t}_{2}\right) \subset N_{2}$

$\Rightarrow \tilde{x}_{e_{1}} \tilde{\in} S S\left(B_{s}\left(\tilde{x}_{e_{1}}, \tilde{r}_{2}, \tilde{t}_{2}\right)\right) \tilde{\subset} S S\left(N_{2}\right)$

$\Rightarrow \tilde{x}_{e_{1}} \tilde{\in} S S\left(\left\{\tilde{z}_{e_{3}}: S\left(\tilde{x}_{e_{1}}, \tilde{z}_{e_{3}}, \tilde{t}_{2}\right) \tilde{>} \overline{1} \ominus \tilde{r_{2}}\right\}\right) \tilde{\subset} S S\left(N_{2}\right)$.

Now take; 
$\tilde{r}(e)=\min \left\{\tilde{r}_{1}(e), \tilde{r}_{2}(e)\right\}, \forall e \in A$,

$\tilde{t}(e)=\max \left\{\tilde{t}_{1}(e), \tilde{t}_{2}(e)\right\}, \forall e \in A$.

Therefore;

$\tilde{x}_{e_{1}} \tilde{\in} S S\left(\left\{\tilde{k}_{e_{4}}: S\left(\tilde{x}_{e_{1}}, \tilde{k}_{e_{4}}, \tilde{t}\right) \tilde{>} \overline{1} \ominus \tilde{r}\right\}\right) \tilde{\subset} S S\left(N_{1}\right) \tilde{\cap} S S\left(N_{2}\right)$

$\Rightarrow S S\left(N_{1}\right) \tilde{\cap} S S\left(N_{2}\right)$ is a soft neighbourhood of $\tilde{x}_{e_{1}}$.

Theorem 3.16. Every soft open ball is a soft neighbourhood of each of its soft points.

Proof: Let $(\tilde{X}, S, \tilde{*})$ be a soft fuzzy metric space. Consider any soft open ball $S S\left(B_{S}\left(\tilde{x}_{e_{1}}, \tilde{r}, \tilde{t}\right)\right.$ with centre $\tilde{x}_{e_{1}}$ and radius $\tilde{r}$ with respect to $\tilde{t}$. From the definition of soft neighbourhood it follows that $S S\left(B_{S}\left(\tilde{x}_{e_{1}}, \tilde{r}, \tilde{t}\right)\right.$ is a soft neighbourhood of $\tilde{x}_{e_{1}}$ in $(\tilde{X}, S, \tilde{*})$. Now take any soft point $\tilde{y}_{e_{2}} \tilde{\in} S S\left(B_{S}\left(\tilde{x}_{e_{1}}, \tilde{r}, \tilde{t}\right)\right.$, other than $\tilde{x}_{e_{1}}$. Then $S\left(\tilde{x}_{e_{1}}, \tilde{y}_{e_{2}}, \tilde{t}\right) \tilde{x}_{1} \ominus \tilde{r}$ and so we can find $\tilde{t} \tilde{>} \tilde{t}_{0} \tilde{>} \overline{0}$ such that $S\left(\tilde{x}_{e_{1}}, \tilde{y}_{e_{2}}, \tilde{t}_{0}\right) \tilde{>} \overline{1} \ominus \tilde{r}$. Let $S\left(\tilde{x}_{e_{1}}, \tilde{y}_{e_{2}}, \tilde{t}_{0}\right)=\tilde{r}_{0} \tilde{>} \overline{1} \ominus \tilde{r}$. Since $\tilde{r}_{0} \tilde{>} \overline{1} \ominus \tilde{r}$ there exist $\exists \tilde{r}_{1} \tilde{\in}(0,1)(E)$ such that $\tilde{r}_{0} \tilde{>} \overline{1} \ominus \tilde{r}_{1} \tilde{>} \overline{1} \ominus \tilde{r}$. For given $\tilde{r}_{0}$ and $\tilde{r}_{1}$ since $\tilde{r}_{0} \tilde{>} \overline{1} \ominus \tilde{r}_{1}$ there exist $\exists \tilde{r}_{2} \tilde{\in}(0,1)(E)$ such that $\tilde{r}_{0} \tilde{*} \tilde{r}_{2} \tilde{\geq} \overline{1} \ominus \tilde{r}_{1}$.

Now consider the soft open ball $S S\left(B_{S}\left(\tilde{y}_{e_{2}}, \overline{1} \ominus \tilde{r}_{2}, \tilde{t} \ominus \tilde{t}_{0}\right)\right)$. We claim that $S S\left(B_{S}\left(\tilde{y}_{e_{2}}, \overline{1} \ominus \tilde{r}_{2}, \tilde{t} \ominus \tilde{t}_{0}\right)\right) \tilde{\subset} S S\left(B S_{S}\left(\tilde{x}_{e_{1}}, \tilde{r}, \tilde{t}\right)\right.$. Take arbitrary $\tilde{z}_{e_{3}} \tilde{\in} S S\left(B_{S}\left(\tilde{y}_{e_{2}}, \overline{1} \ominus \tilde{r}_{2}, \tilde{t} \ominus \tilde{t}_{0}\right)\right)$, then it follows that $S\left(\tilde{y}_{e_{2}}, \tilde{z}_{e_{3}}, \tilde{t} \ominus \tilde{t}_{0}\right) \tilde{>} \tilde{r}_{2}$. By the condition of soft fuzzy metric space (SFM-4)

$$
\begin{gathered}
S\left(\tilde{x}_{e_{1}}, \tilde{z}_{e_{3}}, \tilde{t}\right) \tilde{\geq} S\left(\tilde{x}_{e_{1}}, \tilde{y}_{e_{2}}, \tilde{t}_{0}\right) \tilde{*} S\left(\tilde{y}_{e_{2}}, \tilde{z}_{e_{3}}, \tilde{t} \ominus \tilde{t}_{0}\right) \\
\tilde{>} \tilde{r}_{0} \tilde{*} \tilde{r}_{2} \tilde{\geq} \overline{1} \ominus \tilde{r}_{1} \tilde{>} \overline{1} \ominus \tilde{r} .
\end{gathered}
$$

It means that $\tilde{z}_{e_{3}} \tilde{\in} S S\left(B_{S}\left(\tilde{x}_{e_{1}}, \tilde{r}, \tilde{t}\right)\right.$. We find that; any soft point $\tilde{y}_{e_{2}} \tilde{\in} S S\left(B_{S}\left(\tilde{x}_{e_{1}}, \tilde{r}, \tilde{t}\right)\right.$, other than $\tilde{x}_{e_{1}}$ it can be found a soft open ball $S S\left(B_{S}\left(\tilde{y}_{e_{2}}, \overline{1} \ominus \tilde{r}_{2}, \tilde{t} \ominus \tilde{t}_{0}\right)\right)$ which is $S S\left(B_{S}\left(\tilde{y}_{e_{2}}, \overline{1} \ominus \tilde{r}_{2}, \tilde{t} \ominus \tilde{t}_{0}\right)\right) \tilde{\subset} S S\left(B_{S}\left(\tilde{x}_{e_{1}}, \tilde{r}, \tilde{t}\right)\right.$. And so, because of the definition of soft neighbourhood $S S\left(B_{S}\left(\tilde{x}_{e_{1}}, \tilde{r}, \tilde{t}\right)\right.$ is a soft neighbourhood of each of its point.

Definition 3.17. Let $(Y, A)$ be a soft subset in a soft fuzzy metric space $(\tilde{X}, S, \tilde{*})$. Then $\tilde{x}_{e_{1}}$ is said to be interior point of the soft set $(Y, A)$ if there exist $\tilde{r} \tilde{\in}(0,1)(E)$ and $\tilde{t} \tilde{>} \overline{0}$ such that $\tilde{x}_{e_{1}} \in B_{s}\left(\tilde{x}_{e_{1}}, \tilde{r}, \tilde{t}\right) \subset S P(Y, A)$.

Definition 3.18. Let $(Y, A)$ be a soft subset in a soft fuzzy metric space $(\tilde{X}, S, \tilde{*})$. Then the interior of the soft set $(Y, A)$ is defined to be the set consisting of all interior points of $(Y, A)$.

The interior of the soft set $(Y, A)$ is denoted by $(Y, A)^{\circ}$. Thus,

$(Y, A)^{o}=\left\{\tilde{x}_{e_{1}} \tilde{\in}(Y, A): \tilde{x}_{e_{1}} \in B_{s}\left(\tilde{x}_{e_{1}}, \tilde{r}, \tilde{t}\right) \subset S P(Y, A)\right.$, for some $\tilde{r} \tilde{\in}(0,1)(E)$ and $\left.\tilde{t} \tilde{>} \overline{0}\right\}$.

$\left.S S\left((Y, A)^{\circ}\right)\right)$ is said to be the soft interior of $(Y, A)$.

Theorem 3.19. Let $(Y, A),(Z, A)$ be two non-empty soft subsets in a soft fuzzy metric spaces $(\tilde{X}, S, \tilde{*})$. Then,

(i) $S S\left((Y, A)^{\circ}\right) \tilde{\subset}(Y, A)$

(ii) $(Y, A) \tilde{\subset}(Z, A) \Rightarrow S S\left((Y, A)^{\circ}\right) \tilde{\subset} S S\left((Z, A)^{\circ}\right)$

(iii) $S S\left((Y, A)^{\circ}\right) \tilde{\cap} S S\left((Z, A)^{\circ}\right)=S S\left[((Y, A) \tilde{\cap}(Z, A))^{\circ}\right]$

(iv) $S S\left[((Y, A) \tilde{\cup}(Z, A))^{\circ}\right] \tilde{\supset} S S\left((Y, A)^{\circ}\right) \tilde{\cup} S S\left((Z, A)^{\circ}\right)$

Proof: $(i)$ By the definition of soft interior of $(Y, A),(Y, A)^{\circ} \subset S P(Y, A)$.

Then $S S\left((Y, A)^{\circ}\right) \subset S S(S P(Y, A))=(Y, A)$. (Remember Proposition 1)

Also, (ii) is easy to say that the soft set $S S\left((Y, A)^{\circ}\right)$ is the largest open soft set which is covered by $(Y, A)$.

(ii) Let $(Y, A) \tilde{\subset}(Z, A)$. By (i), $S S\left((Y, A)^{\circ}\right) \tilde{\subset}(Y, A)$ and $S S\left((Z, A)^{\circ}\right) \tilde{\subset}(Z, A)$. Then, we can write $S S\left((Y, A)^{\circ}\right) \tilde{\subset}(Y, A) \tilde{\subset} S S((Z$, and so $S S\left((Y, A)^{\circ}\right) \tilde{\subset} S S\left((Z, A)^{\circ}\right)$.

$($ iii $)$ Let $\tilde{x}_{e_{1}} \tilde{\in} S S\left[((Y, A) \tilde{\cap}(Z, A))^{\circ}\right]$ then there exist $\tilde{r} \tilde{\in}(0,1)(E)$ and $\tilde{t} \tilde{>} \overline{0}$ such that; $\tilde{x}_{e_{1}} \in B_{S}\left(\tilde{x}_{e_{1}}, \tilde{r}, \tilde{t}\right) \subset S P((Y, A) \tilde{\cap}(Z, A))=$ $S P(Y, A) \cap S P(Z, A)$.

$\Rightarrow \tilde{x}_{e_{1}} \in B_{S}\left(\tilde{x}_{e_{1}}, \tilde{r}, \tilde{t}\right) \subset S P(Y, A)$ and $\tilde{x}_{e_{1}} \in B_{S}\left(\tilde{x}_{e_{1}}, \tilde{r}, \tilde{t}\right) \subset S P(Z, A)$

$\Rightarrow \tilde{x}_{e_{1}} \tilde{\in} S S\left((Y, A)^{\circ}\right)$ and $\tilde{x}_{e_{1}} \tilde{\in} S S\left((Z, A)^{\circ}\right)$

$\Rightarrow \tilde{x}_{e_{1}} \tilde{\in} S S\left((Y, A)^{\circ}\right) \tilde{\cap} S S\left((Z, A)^{\circ}\right)$

$$
\Rightarrow S S\left[((Y, A) \tilde{\cap}(Z, A))^{\circ}\right] \tilde{C} S S\left((Y, A)^{\circ}\right) \tilde{\cap} S S\left((Z, A)^{\circ}\right)
$$


Conversely, let $\tilde{y}_{e_{2}} \tilde{E} S S\left((Y, A)^{\circ}\right) \tilde{\cap} S S\left((Z, A)^{\circ}\right)$

$\Rightarrow \tilde{y}_{e_{2}} \tilde{\in} S S\left((Y, A)^{\circ}\right)$ and $\tilde{y}_{e_{2}} \tilde{\in} S S\left((Z, A)^{\circ}\right)$

$\Rightarrow \exists \tilde{r}_{1}, \tilde{r}_{2} \tilde{\in}(0,1)(E)$ and $\tilde{t}_{1}, \tilde{t}_{2} \tilde{>} \overline{0}$ such that $\tilde{y}_{e_{2}} \in B_{S}\left(\tilde{y}_{e_{2}}, \tilde{r}_{1}, \tilde{t}_{1}\right) \subset S P(Y, A)$ and $\tilde{y}_{e_{2}} \in B_{S}\left(\tilde{y}_{e_{2}}, \tilde{r}_{2}, \tilde{t}_{2}\right) \subset$ $S P(Z, A)$. Now take;

$$
\begin{aligned}
& \tilde{r}(e)=\min \left\{\tilde{r}_{1}(e), \tilde{r}_{2}(e)\right\}, \forall e \in A \\
& \tilde{t}(e)=\max \left\{\tilde{t}_{1}(e), \tilde{t}_{2}(e)\right\}, \forall e \in A .
\end{aligned}
$$

So, $\tilde{y}_{e_{2}} \in B_{S}\left(\tilde{y}_{e_{2}}, \tilde{r}, \tilde{t}\right) \subset S P(Y, A)$ and $\tilde{y}_{e_{2}} \in B_{S}\left(\tilde{y}_{e_{2}}, \tilde{r}, \tilde{t}\right) \subset S P(Z, A)$

$\Rightarrow \tilde{y}_{e_{2}} \in B_{S}\left(\tilde{y}_{e_{2}}, \tilde{r}, \tilde{t}\right) \subset S P(Y, A) \cap S P(Z, A)=S P[(Y, A) \tilde{\cap}(Z, A)]$

$\Rightarrow \tilde{y}_{e_{2}} \tilde{\in} S S\left[((Y, A) \tilde{\cap}(Z, A))^{\circ}\right]$

$$
\Rightarrow S S\left((Y, A)^{\circ}\right) \tilde{\cap} S S\left((Z, A)^{\circ}\right) \tilde{C} S S\left[((Y, A) \tilde{\cap}(Z, A))^{\circ}\right]
$$

From (3.1) and (3.2),

$$
S S\left((Y, A)^{\circ}\right) \tilde{\cap} S S\left((Z, A)^{\circ}\right)=S S\left[((Y, A) \tilde{\cap}(Z, A))^{\circ}\right] .
$$

(iv) It is obvious.

Definition 3.20. Let $(\tilde{X}, S, \tilde{*})$ be a soft fuzyy metric space and $(Y, A)$ be a non-empty soft subset of $\tilde{X}$ in $(\tilde{X}, S, \tilde{*})$. Then $(Y, A)$ is said to be soft open in $\tilde{X}$ with respect to $S$ if and only if all soft points of $(Y, A)$ be interior points of $(Y, A)$.

Theorem 3.21. Every soft open ball is a soft open set.

Proof: Consider a soft open ball $S S\left(B_{S}\left(\tilde{x}_{e_{1}}, \tilde{r}, \tilde{t}\right)\right)$ and take $\tilde{y}_{e_{2}} \tilde{\in} S S\left(B_{S}\left(\tilde{x}_{e_{1}}, \tilde{r}, \tilde{t}\right)\right)$. This implies that $S\left(\tilde{x}_{e_{1}}, \tilde{y}_{e_{2}}, \tilde{t}\right) \tilde{>} \overline{1} \ominus$ $\tilde{r}$. Since $S\left(\tilde{x}_{e_{1}}, \tilde{y}_{e_{2}}, \tilde{t}\right) \tilde{>} \overline{1} \ominus \tilde{r}$, we can find a $\tilde{t}_{0}, \overline{0} \tilde{<} \tilde{t}_{0} \tilde{<} \tilde{t}$ such that $S\left(\tilde{x}_{e_{1}}, \tilde{y}_{e_{2}}, \tilde{t}_{0}\right) \tilde{>} \overline{1} \ominus \tilde{r}$. Choose $\tilde{r}_{0}=S\left(\tilde{x}_{e_{1}}, \tilde{y}_{e_{2}}, \tilde{t}_{0}\right) \tilde{>} \overline{1} \ominus$ $\tilde{r}$. Since $\tilde{r}_{0} \tilde{>} \overline{1} \ominus \tilde{r}$ we can find a $\tilde{s} \tilde{\in}(0,1)(E)$ such that $\tilde{r}_{0} \tilde{>} \overline{1} \ominus \tilde{s} \tilde{>} \overline{1} \ominus \tilde{r}$. For a given $\tilde{r}_{0}$ and $\tilde{s}$ such that $\tilde{r}_{0} \tilde{>} \overline{1} \ominus \tilde{s}$, by Remark (3) we can find $\tilde{r}_{1} \tilde{\in}(0,1)(E)$ such that $\tilde{r}_{0} \tilde{*} \tilde{r}_{1} \tilde{\geq} \overline{1} \ominus \tilde{s}$.

Now consider the soft open ball $S S\left(B_{S}\left(\tilde{y}_{e_{2}}, \overline{1} \ominus \tilde{r}_{1}, \tilde{t} \ominus \tilde{t}_{0}\right)\right)$. We claim that

$$
S S\left(B_{S}\left(\tilde{y}_{e_{2}}, \overline{1} \ominus \tilde{r}_{1}, \tilde{t} \ominus \tilde{t}_{0}\right)\right) \tilde{\subset} S S\left(B_{S}\left(\tilde{x}_{e_{1}}, \tilde{r}, \tilde{t}\right)\right) .
$$

Take $\tilde{z}_{e_{3}} \tilde{\in} S S\left(B_{S}\left(\tilde{y}_{e_{2}}, \overline{1} \ominus \tilde{r}_{1}, \tilde{t} \ominus \tilde{t}_{0}\right)\right)$. This implies that $S\left(\tilde{y}_{e_{2}}, \tilde{z}_{e_{3}}, \tilde{t} \ominus \tilde{t}_{0}\right) \tilde{>} \tilde{r}_{1}$. Thus from the triangle inequality (condition SFM-4) for soft fuzzy metric,

$$
\begin{aligned}
S\left(\tilde{x}_{e_{1}}, \tilde{z}_{e_{3}}, \tilde{t}\right) & \tilde{\geq} S\left(\tilde{x}_{e_{1}}, \tilde{y}_{e_{2}}, \tilde{t}_{0}\right) \tilde{*} S\left(\tilde{y}_{e_{2}}, \tilde{z}_{e_{3}}, \tilde{t} \ominus \tilde{t}_{0}\right) \\
& \tilde{\geq} \tilde{r}_{0} \tilde{*}_{\tilde{r}_{1}} \tilde{\geq} \ominus \tilde{1} \ominus \tilde{s} \\
& \tilde{>} \overline{1} \ominus \tilde{r} .
\end{aligned}
$$

Therefore $\tilde{z}_{e_{3}} \tilde{\in} S S\left(B_{S}\left(\tilde{x}_{e_{1}}, \tilde{r}, \tilde{t}\right)\right)$ and since $\tilde{z}_{e_{3}}$ is arbitrary,

$$
S S\left(B_{S}\left(\tilde{y}_{e_{2}}, \overline{1} \ominus \tilde{r}_{1}, \tilde{t} \ominus \tilde{t}_{0}\right)\right) \tilde{C} S S\left(B_{S}\left(\tilde{x}_{e_{1}}, \tilde{r}, \tilde{t}\right)\right) .
$$

Theorem 3.22. Let $(\tilde{X}, S, \tilde{*})$ be a soft fuzzy metric space. Define

$$
\tau_{S}=\left\{(Y, A) \tilde{\subset} \tilde{X}: \forall \tilde{x}_{e_{1}} \tilde{\in}(Y, A), \exists \tilde{t} \tilde{>} \overline{0} \text { and } \tilde{r} \tilde{\in}(0,1)(E) \text { such that } B_{S}\left(\tilde{x}_{e_{1}}, \tilde{r}, \tilde{t}\right)\right.
$$

$$
\subset S P(Y, A)\} \text {. }
$$

Then $\tau_{S}$ is a topology on $\tilde{X}$. 
Proof:

$\left(t_{1}\right)$ It is clear that $\Phi$ and $\tilde{X}$ belong to $\tau_{S}$.

$\left(t_{2}\right)$ Let $I$ be an arbitrary index set and the soft sets $\left(Y_{i}, A\right) \tilde{\subset} \tilde{X}$ be soft opens in $(\tilde{X}, S, \tilde{*})$, for all $i \in I$. We need to show that $\tilde{U}_{i \in I}\left(Y_{i}, A\right)$ is also soft open in $(\tilde{X}, S, \tilde{*})$.

(i) If $\tilde{U}_{i \in I}\left(Y_{i}, A\right)=\tilde{X}$, then by $\left(t_{1}\right) \tilde{X}$ belongs to $\tau_{S}$.

(ii) Let $\tilde{\bigcup}_{i \in I}\left(Y_{i}, A\right) \neq \Phi$ and take $\tilde{x}_{e_{1}} \tilde{\in} \tilde{\bigcup}_{i \in I}\left(Y_{i}, A\right)$. In this case $\tilde{x}_{e_{1}} \tilde{\in}\left(Y_{i}, A\right)$ for some $i \in I$. Since each $\left(Y_{i}, A\right)$ is soft open in $(\tilde{X}, S, \tilde{*})$, there exist $\tilde{r} \tilde{\in}(0,1)(E)$ and $\tilde{t} \tilde{>} \overline{0}$ such that; $\tilde{x}_{e_{1}} \in B_{S}\left(\tilde{x}_{e_{1}}, \tilde{r}, \tilde{t}\right) \subset S P\left(Y_{i}, A\right) \subset S P\left(\tilde{U}\left(Y_{i}, A\right)\right)$. Then $\tilde{U}_{i \in I}\left(Y_{i}, A\right)$ is soft open in $(\tilde{X}, S, \tilde{*})$.

$\left(t_{3}\right)$ Let $I=\{1,2, \ldots, n\}$ be index set and the soft $\operatorname{sets}\left(Y_{i}, A\right) \tilde{\subset} \tilde{X}$ be soft opens in $(\tilde{X}, S, \tilde{*})$, for all $i \in I$. We need to show that $\bigcap_{i=1}^{n}\left(Y_{i}, A\right)$ is also soft open in $(\tilde{X}, S, \tilde{*})$.

(i) If $\overbrace{i=1}^{n}\left(Y_{i}, A\right)=\Phi$ then by $\left(t_{1}\right) \Phi$ belongs to $\tau_{S}$.

(ii) Let $\bigcap_{i=1}^{n}\left(Y_{i}, A\right) \neq \Phi$ and take $\tilde{x}_{e_{1}} \tilde{\in} \overbrace{i=1}^{n}\left(Y_{i}, A\right)$, then $\tilde{x}_{e_{1}} \tilde{\in}\left(Y_{i}, A\right)$ for all $i=\{1,2, \ldots, n\}$. Hence for each $i=$ $\{1,2, \ldots, n\}$, there exist $\tilde{t} \tilde{>} \overline{0}$ and $\tilde{r} \tilde{\epsilon}(0,1)(E)$ such that $\tilde{x}_{e_{1}} \in B_{S}\left(\tilde{x}_{e_{1}}, \tilde{r}_{i}, \tilde{t}_{i}\right) \subset S P\left(Y_{i}, A\right)$. Take

$$
\begin{aligned}
& \tilde{r}(e)=\min \left\{\tilde{r}_{i}(e): i=1,2, \ldots, n\right\}, e \in A \\
& \tilde{t}(e)=\max \left\{\tilde{t}_{i}(e): i=1,2, \ldots, n\right\}, e \in A .
\end{aligned}
$$

Then for $i=\{1,2, \ldots, n\}, \tilde{x}_{e_{1}} \in B_{S}\left(\tilde{x}_{e_{1}}, \tilde{r}, \tilde{t}\right) \subset \bigcap_{i=1}^{n} S P\left(Y_{i}, A\right)$. So $\tilde{x}_{e_{1}} \in B_{S}\left(\tilde{x}_{e_{1}}, \tilde{r}, \tilde{t}\right) \subset \bigcap_{i=1}^{n} S P\left(Y_{i}, A\right)=S P\left(\bigcap_{i=1}^{n}\left(Y_{i}, A\right)\right)$ and $\bigcap_{i=1}^{n}\left(Y_{i}, A\right)$ is soft open.

Definition 3.23. Let $(\tilde{X}, S, \tilde{*})$ be a soft fuzyy metric space. A soft set $(Y, A) \tilde{C} \tilde{X}$, is said to be soft closed in $\tilde{X}$ with respect to $S$ if its complement $(Y, A)^{c}=\left(Y^{c}, A\right)$ is soft open in $(\tilde{X}, S, \tilde{*})$.

Theorem 3.24. In any soft fuzzy metric space $(\tilde{X}, S, \tilde{*})$,

(i) $\Phi$ and $\tilde{X}$ are soft closed;

(ii) Arbitary intersection of soft closed sets is soft closed;

(iii) Union of a finite number of closed sets is closed.

Proof: $(i)$ From Theorem 2, $\Phi$ and $\tilde{X}$ is soft open. Then $(\Phi, A)^{c}=\left(\Phi^{c}, A\right)=(\tilde{X}, A)$ and $(\tilde{X}, A)^{c}=\left(\tilde{X}^{c}, A\right)=$ $(\Phi, A)$ are soft closed.

(ii) Let $I$ be an arbitary indexed set and the soft sets $\left(F_{i}, A\right) \tilde{C} \tilde{X}$ be soft closed in $(\tilde{X}, S, \tilde{*})$, for all $i \in I$. We need to show that $\tilde{\cap}_{i \in I}\left(F_{i}, A\right)$ is also soft closed in $(\tilde{X}, S, \tilde{*})$.

Since $\left(F_{i}, A\right)$ is soft closed for all $i \in I$, then $\left(F_{i}, A\right)^{c}=\left(F_{i}^{c}, A\right)$ is soft open in $(\tilde{X}, S, \tilde{*})$, for all $i \in I$. By the Theorem 2-( $\left.t_{2}\right) \underset{i \in I}{\tilde{U}}\left(F_{i}^{c}, A\right)$ is soft open in $(\tilde{X}, S, \tilde{*})$.

Then $\tilde{\cap}_{i \in I}\left(F_{i}, A\right)$ is soft closed for all $i \in I$. 
(iii) Let $I=\{1,2, \ldots, n\}$ be indexed set and the soft sets $\left(F_{i}, A\right) \tilde{\subset} \tilde{X}$ be soft closed in $(\tilde{X}, S, \tilde{*})$, for all $i \in I$. We need to show that $\underset{i=1}{\tilde{U}}\left(F_{i}, A\right)$ is also soft closed for all $i \in I$.

$\left[\tilde{\cup}_{i=1}^{n}\left(F_{i}, A\right)\right]^{c}=\left[\tilde{\cap}_{i=1}^{n}\left(F_{i}^{c}, A\right)\right]$, for all $i \in I$.

Since $\left(F_{i}, A\right)$ is soft closed then $\left(F_{i}^{c}, A\right)$ is soft open for all $i=\{1,2, \ldots, n\}$. By Theorem $2-\left(t_{3}\right), \underset{i=1}{\stackrel{n}{\sim}}\left(F_{i}^{c}, A\right)$ is soft open in $(\tilde{X}, S, \tilde{*})$. Then $\underset{i=1}{\stackrel{n}{U}}\left(F_{i}, A\right)$ is soft closed for all $i=\{1,2, \ldots, n\}$.

Definition 3.25. Let $(\tilde{X}, S, \tilde{*})$ be a soft fuzzy metric space and $(Y, A) \tilde{\subset} \tilde{X}$. A soft point $\tilde{x}_{e_{1}} \tilde{\in} \tilde{X}$ is said to be a soft cluster point of $(Y, A)$, if and only if every soft open ball $S S\left(B_{s}\left(\tilde{x}_{e_{1}}, \tilde{r}, \tilde{t}\right)\right)$ containing $\tilde{x}_{e_{1}}$ in $(\tilde{X}, S, \tilde{*})$ consist at least one soft point of $(Y, A)$, other then $\tilde{x}_{e_{1}}$.

A soft cluster point of soft set $(Y, A)$ may or may not belong to the soft set $(Y, A)$.

Definition 3.26. Let $(\tilde{X}, S, \tilde{*})$ be a soft fuzzy metric space and $(Y, A) \tilde{\subset} \tilde{X}$. Then the soft set generated by the collection of all soft points of $(Y, A)$ and soft cluster points of $(Y, A)$ in $(\tilde{X}, S, \tilde{*})$ is said to be soft closure of $(Y, A)$ in $(\tilde{X}, S, \tilde{*})$. It is denoted by $\overline{(Y, A)}$.

\section{Conclusion}

In this paper, we introduced soft fuzzy metric which is different from the definition of fuzzy soft metric in [4] and [5]. We examined some topological structures in this new space. Our results can be improved in theoretical mathematics and computer sciences.

ACKNOWLEDGEMENTS. We thank the edit $\tilde{A} \uparrow \mathrm{r}$ and referee for their comments.

\section{References}

[1] K. T. Atanassov, Intuitionistic fuzzy sets, Fuzzy Sets and Systems, 20(1), (1986), 87-96.

[2] K. T. Atanassov, Operators over interval valued intuitionistic fuzzy sets, Fuzzy Sets and Systems, 64(2), (1994), 159-174.

[3] A. Aygnolu, Soft Topological Spaces, Ph.D Thesis, The Graduate School of Natural and Applied Science of Kocaeli University. (2011).

[4] T. Beaula and C. Gunaseeli, C. On fuzzy soft metric spaces, Malaya Journal of Mathematics, 2(3), (2014), 197-202.

[5] T. Beaula and M. M. Priyanga, A new notion for fuzzy soft normed space, International Journal of Fuzzy Mathematical Archive, 9(1), (2015), 81-90.

[6] S. Das and S. K. Samanta, Soft metric, Ann. Fuzzy Math. Inform., 6(1), (2013), 77-94.

[7] S. Das and S. K. Samanta, Soft real sets, soft real numbers and their properties, J. Fuzzy Math., 20(3), (2012), 551-576.

[8] S. Das and S. K. Samanta, On soft metric spaces, J. Fuzzy Math., 21(3), (2013), 707-734.

[9] A. C. Güler, E. D. Yıldırım and O. B. Ozbakır, A fixed point theorem on soft G-metric spaces, J. Nonlinear Sci. Appl., 9(3), (2016), 885-894.

[10] P. K. Maji, A. Roy and R. Biswas, An application of soft sets in a decision making problem, Computers Math. Appl., 44, (2002), 1077-1083.

[11] P. K. Maji, R. Biswas and A. Roy, Soft set theory, Computers 85 Mathematics with Applications, 45(4-5), (2003), 555-562. 
[12] D. Molodtsov, Soft set theory-First results, Computers \& Mathematics with Applications, 37(4-5), (1999), 19-31.

[13] B. Schweizer and A. Sklar, Statistical metric spaces, Pacific J. Math., 10(1), (1960), 313-334.

[14] M. Shabir, and M. Naz, On soft topological spaces , Computers \& Mathematics with Applications, 61(7), (2011), 1786-1799.

[15] O. A. Tantawy and R. M. Hassan, Soft real analysis , Journal of Prograssive in Mathematics, 8(1), (2016), 1207-1219.

[16] L.A. Zadeh, Fuzzy sets, Information and Control, 8(3), (1965), 338-353. 